\title{
A randomized phase III trial of personalized peptide vaccination for castration-resistant prostate cancer progressing after docetaxel
}

\author{
MASANORI NOGUCHI ${ }^{1}$, KIYOHIDE FUJIMOTO ${ }^{2}$, GAKU ARAI $^{3}$, HIROJI UEMURA $^{4}$, KATSUYOSHI HASHINE ${ }^{5}$, \\ HIROAKI MATSUMOTO $^{6}$, SATOSHI FUKASAWA ${ }^{7}$, YASUO KOHJIMOTO ${ }^{8}$, HIDEOMI NAKATSU $^{9}$, \\ ATSUSHI TAKENAKA ${ }^{10}$, MASATO FUJISAWA ${ }^{11}$, HIROTSUGU UEMURA ${ }^{12}$, SEIJI NAITO $^{13}$, \\ SHIN EGAWA ${ }^{14}$, HIROYUKI FUJIMOTO ${ }^{15}$, SHIRO HINOTSU ${ }^{16}$ and KYOGO ITOH ${ }^{1}$ \\ ${ }^{1}$ Cancer Vaccine Center and Department of Urology, Kurume University, Kurume, Fukuoka 839-0863; \\ ${ }^{2}$ Department of Urology, Nara Medical University Hospital, Kashihara, Nara 634-8522; ${ }^{3}$ Department of Urology, \\ Dokkyo Medical University Saitama Medical Center, Koshigaya, Saitama 343-8555; ${ }^{4}$ Department of Urology, \\ Yokohama City University Medical Center, Yokohama, Kanagawa 232-0024; ${ }^{5}$ Department of Urology, Shikoku Cancer Center, \\ Matsuyama, Ehime 791-0280; ${ }^{6}$ Department of Urology, Yamaguchi University Hospital, Ube, Yamaguchi 755-8505; \\ ${ }^{7}$ Department of Urology, Chiba Cancer Center, Chiba, Chiba 260-8717; ${ }^{8}$ Department of Urology, \\ Wakayama Medical University Hospital, Wakayama, Wakayama 641-8510; ${ }^{9}$ Department of Urology, Asahi General Hospital, \\ Asahi, Chiba 289-2511; ${ }^{10}$ Department of Urology, Tottori University Hospital, Yonago, Tottori 683-8504; \\ ${ }^{11}$ Department of Urology, Kobe University Hospital, Kobe, Hyogo 650-0017; ${ }^{12}$ Department of Urology, \\ Kindai University Hospital, Sayama, Osaka 589-8511; ${ }^{13}$ Department of Urology, Sanshinkai Hara Hospital, \\ Fukuoka, Fukuoka 812-0033; ${ }^{14}$ Department of Urology, The Jikei University Hospital, Minatoku, Tokyo 105-8471; \\ ${ }^{15}$ Department of Urology, National Cancer Center Hospital, Chyuoku, Tokyo 104-0045; \\ ${ }^{16}$ Department of Urology, Sapporo Medical University, Sapporo, Hokkaido 060-8543, Japan
}

Received June 6, 2020; Accepted October 13, 2020

DOI: $10.3892 / o r .2020 .7847$

\begin{abstract}
First-line chemotherapy for men with metastatic castration-resistant prostate cancer (mCRPC) has been employed to improve overall survival (OS) and progression-free survival (PFS). However, several new agents for CRPC after first-line chemotherapy prolonged survival by only a few months. To develop a new treatment modality, we conducted a phase III randomized trial of personalized peptide vaccination (PPV) for human leukocyte
\end{abstract}

Correspondence to: Dr Masanori Noguchi, Cancer Vaccine Center and Department of Urology, Kurume University, 155-1 Kokubu-machi, Kurume, Fukuoka 839-0863, Japan

E-mail:nogu-masa@tkz.bbiq.jp

Abbreviations: AEs, adverse events; CRPC, castration-resistant prostate cancer; CTL, cytotoxic T lymphocytes; ECOG, Eastern Cooperative Oncology Group; HLA, human leukocyte antigen; IgG, immunoglobulin G; MDSC, myeloid-derived suppressor cells; NLR, neutrophil to lymphocyte ratio; OS, overall survival; PD, progressive disease; PFS, progression-free survival; PPV, personalized peptide vaccination; PSA, prostate-specific antigen

Key words: prostate cancer, cancer vaccine, multiple-peptide vaccine, immunotherapy, docetaxel antigen (HLA)-A24-positive patients with castration-resistant prostate cancer (CRPC) for whom docetaxel chemotherapy failed. This randomized, double-blind, placebo-controlled, phase III trial was carried out at 68 medical centers in Japan. Patients were randomly assigned at a 2:1 ratio to receive PPV or placebo. Four of 12 warehouse peptides selected based on pre-existing peptide-specific immunoglobulin $G$ levels or the corresponding placebo were subcutaneously injected in 6 doses weekly and then bi-weekly following the maximum of 30 doses until disease progression. The primary end-point was overall survival (OS). Efficacy analyses were performed by the full analysis set. Between August 2013 and April 2016, 310 patients were randomly assigned, and 306 patients were analyzed. Baseline characteristics were balanced between groups. The estimated median OS was 16.1 months [95\% confidence interval (CI), 13-18.2] with PPV and 16.9 months (95\% CI, 13.1-20.4) with placebo [hazard ratio (HR), 1.04, 95\% CI, 0.80-1.37; $\mathrm{P}=0.77]$. Grade $\geq 3$ adverse events were observed in $41 \%$ of both groups. The analysis of treatment arm effects among subgroups revealed lower HRs for OS in favor of the PPV arm in patients with $<64 \%$ neutrophils (HR, 0.55 , 95\% CI, 0.33-0.93; $\mathrm{P}=0.03$ ) or $\geq 26 \%$ lymphocytes (HR, 0.70 , 95\% CI, 0.52-0.92; $\mathrm{P}=0.02$ ) at baseline. PPV did not prolong OS in HLA-A24-positive patients with CRPC progressing after docetaxel chemotherapy. Subgroup analysis suggested that the patients with a lower proportion of neutrophils or a higher proportion of lymphocytes at baseline can receive survival benefits from PPV treatment. 


\section{Introduction}

Since 2004, docetaxel has been the standard first-line chemotherapy for men with metastatic castration-resistant prostate cancer (mCRPC) based on improvements in overall survival (OS) and progression-free survival (PFS) compared with mitoxantrone and prednisone $(1,2)$. Although most patients receive docetaxel chemotherapy for mCRPC, the cancer will eventually progress and no consensus exists for the optimal intervention after docetaxel failure. The US Food and Drug Administration (FDA) approved several new drugs for patients with mCRPC for whom docetaxel chemotherapy failed, including cabazitaxel (3), abiraterone with prednisone (4), enzalutamide (5), and radium-223 (6). Although these advancements have been made, the improvement in survival by these drugs is only several months and mCRPC continues to be incurable. Therefore, treatments that can provide stable disease control and long-term survival benefits are needed.

In the last few decades, immunotherapy has become an important part of treating several types of cancer. Sipuleucel-T is currently the only approved cellular product immune therapy for the treatment of asymptomatic or minimally symptomatic CRPC (7). Although immune checkpoint inhibitors, including cytotoxic T-lymphocyte antigen-4 (CTLA-4), programmed death 1 (PD1), and programmed death-ligand 1 (PD-L1) were recently approved by the FDA to treat different types of solid tumors and hematologic malignancies, none of these immune checkpoint inhibitors have been approved for mCRPC $(8,9)$. In addition, neither peptide-based vaccine trials nor recent conducted immunotherapy studies demonstrated clinical benefits for mCRPC in large randomized trials (10). This failure may be due to the large diversity of immunological features of mCRPC patients and the lack of readily available biomarkers of immunotherapy benefits.

To overcome these difficulties, we are developing a new concept of personalized peptide vaccination (PPV) for patients with advanced cancer, in which up to 4 peptides are selected from a collection of warehouse peptides based on pre-existing immunity (11). A phase I and follow-up study of PPV consisting of 14 warehouse peptides for human leukocyte antigen (HLA) -A24-positive patients with advanced CRPC demonstrated its safety and potential clinical benefit (12). A randomized phase II trial of PPV with low-dose dexamethasone for patients with chemotherapy-naïve CRPC also resulted in a longer PFS of prostate-specific antigen (PSA) and OS (13). In addition, another phase II study suggested that the OS of docetaxel-resistant CRPC patients treated with PPV was longer than that of historical controls (14).

Based on these results, a randomized, double-blind, placebo-controlled, phase III trial of PPV for HLA-A24-positive patients with CRPC progressing after docetaxel chemotherapy with OS as the primary endpoint was conducted in Japan.

\section{Materials and methods}

Patient population. For this phase III, randomized, double-blind, placebo-controlled study, we enrolled HLA-A24-positive patients with CRPC progressing within 12 months after docetaxel chemotherapy from 68 medical centers in Japan. Eligible patients were aged 20 years or older with histologically confirmed adenocarcinoma of the prostate. Other inclusion criteria were as follows: positive immunoglobulin $\mathrm{G}(\mathrm{IgG})$ responses to at least 2 of 12 warehouse peptides (Table SI) on the screening test, an Eastern Cooperative Oncology Group (ECOG) performance status (PS) of 0 or 1 , life expectancy of $\geq 12$ weeks, serum testosterone level of $\leq 50 \mathrm{ng} / \mathrm{dl}$, and satisfactory bone marrow function, hepatic function, and renal function. Patients without previous bilateral orchiectomy continued treatment with luteinizing hormone-releasing agonists. Exclusion criteria included acute infection, history of severe allergic reactions, pulmonary, cardiac or other systemic diseases, or other inappropriate conditions for enrollment as determined by the clinicians. Prior enzalutamide or abiraterone was permitted.

The trial was conducted in accordance with the Declaration of Helsinki and Good Clinical Practice guidelines. The protocol was approved by institutional review boards or ethical committees at all of the institutions, and it was registered in the UMIN Clinical Trials Registry (no. UMIN000011308). All patients were Japanese and provided written informed consent before participating in this study.

Study design and treatment. Patients were randomly assigned at a 2:1 ratio to receive PPV or placebo using the minimization technique with the following stratification factors: age ( $<75$ or $\geq 75$, PS ( 0 or 1 ), and use of enzalutamide or abiraterone (with or without) at each participating institution. The present study was double-blinded, and all physicians, patients, and investigators providing the interventions, assessing outcomes, and analyzing data were blinded to treatment assignment. Up to 4 of 12 warehouse peptides selected based on pre-existing peptide-specific IgG levels or the corresponding placebos were emulsified with Montanide ISA 51 incomplete Freund's adjuvant (Seppic), and each study drug (up to 4 ) in a $1.5-\mathrm{ml}$ emulsion ( $3 \mathrm{mg} /$ peptide or saline solution) was subcutaneously injected in 6 doses weekly and then bi-weekly following the maximum of 30 doses until disease progression.

Outcomes. The primary end point was OS, which was defined as the time from random assignment to death by any cause. Secondary end points were PFS, one-year survival rate, immune responses, and safety. PFS was defined as the time from random assignment until objective disease progression based on the PSA Working Group Consensus Criteria 2 (PCWG2), the Response Evaluation Criteria in Solid Tumors (RECIST) 1.1 criteria, or death. Immune responses were assessed by IgG titers measured by the Luminex system (15) and cytotoxic T lymphocyte (CTL) activity measured by the interferon (IFN)- $\gamma$ release assay (16) using blood sampled at pre-treatment and every 6 treatments. Safety was assessed based on physical examination, vital sign measurements, clinical laboratory analyses, and adverse events (AEs) graded using the Common Terminology Criteria for Adverse Events (CTCAE) version 4.0.

Statistical analysis. All efficacy analyses were based on the full analysis set (FAS), defined as patients who received at least one dose of the treatment. The planned sample size of 300 patients (200 in PPV and 100 in placebo arms) provided $90 \%$ of power at a two-sided significance level of 0.05 to 


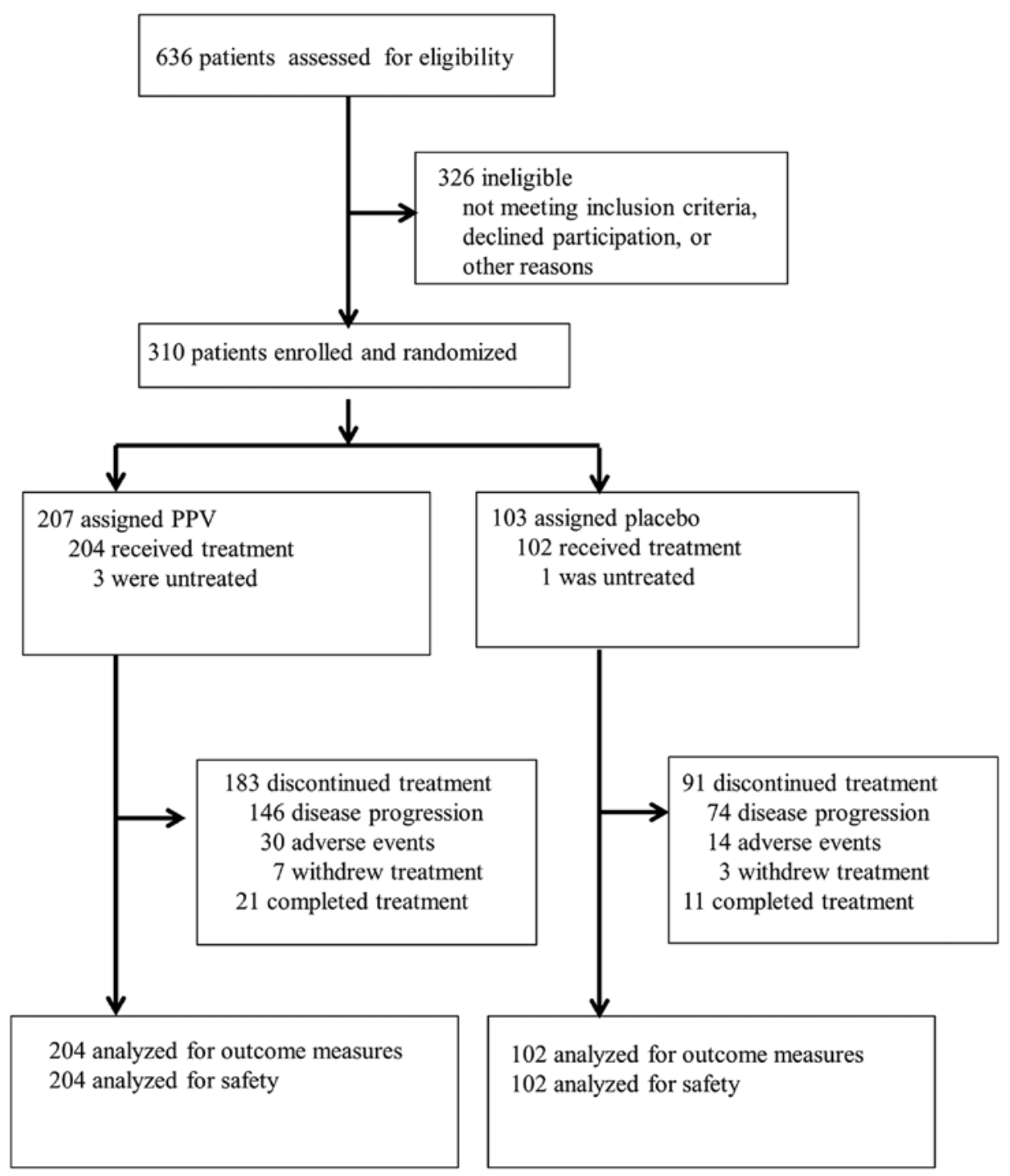

Figure 1. Patient flowchart. PPV, personalized peptide vaccination.

detect a hazard ratio (HR) of 0.6 for the primary endpoint of OS, corresponding to an increase in the median OS from 11 to 17 months. All analyses were performed after 23 months from the last patient enrolled or when $80 \%$ of events for the primary endpoint of OS was reached. The follow-up ended in October 2017 and final analyses were performed using a database adjusted to May 2018.

Survival curves were described according to the Kaplan-Meier method and 95\% confidence intervals (CIs) were calculated. Comparison of OS was performed by the Harrington-Fleming test. A log-rank test using stratified randomization was used to compare OS for PPV versus placebo. The effects of treatments were reported as HRs, 95\% CIs, and interaction $\mathrm{P}$ for subgroup categories in a Forest plot. The Student's t-test was used to compare quantitative variables. The mean change from baseline in immune responses at each time point was compared between the two arms in a linear regression model. All toxicity grades and severe (grade $\geq 3$ ) toxicities were compared between the two arms (Fisher's exact test). All statistical tests were interpreted as significant with a P-value of $<5 \%$. All analyses were performed using JMP version 13 (SAS Institution).

\section{Results}

Patient characteristics. Between August 2013 and April 2016, 636 patients were screened for eligibility in this study, and 310 eligible patients were randomly assigned to the PPV arm $(n=207)$ or placebo arm $(n=103)$. The most common reason for ineligibility was HLA mismatch. Three patients in the PPV arm and 1 in the placebo arm did not receive treatment, and 306 patients were analyzed by the full analysis set (204 for PPV and 102 for placebo). A patient flowchart is shown in Fig. 1. No imbalances existed between randomization arms except for a lower PSA level in the PPV arm (Table I).

Efficacy. At the final analysis (cut-off date: October 1, 2017), the median length of follow-up for censored patients was 29.8 months in the PPV arm and 27.4 months in the placebo arm, and $89.5 \%$ of patients had discontinued treatment. The median total number of doses of treatment drugs was 12 (IQR, 8 to 19) in the PPV arm and 14 (IQR, 10 to 21) in the placebo arm. The reasons for discontinuation (PPV vs. placebo) were disease progression (71.6 vs. $72.5 \%$ ), AEs (14.7 vs. $13.7 \%$ ) or 
Table I. Baseline patient characteristics by treatment arm.

\begin{tabular}{|c|c|c|c|c|}
\hline \multirow[b]{2}{*}{ Characteristics } & \multicolumn{2}{|c|}{$\operatorname{PPV}(n=204)$} & \multicolumn{2}{|c|}{ Placebo $(n=102)$} \\
\hline & No. & $\%$ & No. & $\%$ \\
\hline Median age, years (range) & \multicolumn{2}{|c|}{$71.0(53-84)$} & \multicolumn{2}{|c|}{$72.0(56-82)$} \\
\hline \multicolumn{5}{|l|}{ Age group (years) } \\
\hline$<65$ & 37 & 18.1 & 19 & 18.6 \\
\hline $66-74$ & 104 & 51 & 52 & 51 \\
\hline$\geq 75$ & 63 & 30.9 & 31 & 30.4 \\
\hline \multicolumn{5}{|l|}{ ECOG performance status } \\
\hline 0 & 162 & 79.4 & 82 & 80.4 \\
\hline 1 & 42 & 20.6 & 20 & 19.6 \\
\hline \multicolumn{5}{|c|}{ Prior use of enzalutamide or abiraterone } \\
\hline Yes & 66 & 32.4 & 35 & 34.3 \\
\hline No & 44 & 21.6 & 20 & 19.6 \\
\hline Unknown & 94 & 46 & 47 & 46.1 \\
\hline \multicolumn{5}{|l|}{ Serum PSA level, ng/ml } \\
\hline$<50$ & 109 & 53.4 & 50 & 49 \\
\hline $50-499$ & 83 & 40.7 & 37 & 36.3 \\
\hline$\geq 500$ & 12 & 5.9 & 15 & 14.7 \\
\hline \multicolumn{5}{|l|}{ Gleason score at diagnosis } \\
\hline$<6$ & 4 & 2.4 & 3 & 2.9 \\
\hline 7 & 30 & 14.6 & 20 & 19.6 \\
\hline$\geq 8$ & 166 & 80.6 & 75 & 73.5 \\
\hline Unknown & 4 & 2.4 & 4 & 4 \\
\hline \multicolumn{5}{|l|}{ No. of metastatic sites } \\
\hline 0 & 15 & 7.3 & 7 & 6.9 \\
\hline 1 & 115 & 56.4 & 61 & 59.8 \\
\hline$\geq 2$ & 74 & 36.3 & 34 & 33.3 \\
\hline \multicolumn{5}{|c|}{ Median proportion of WBC type, $\%$ (range) } \\
\hline Neutrophils & \multicolumn{2}{|c|}{$70.0(35-94.1)$} & \multicolumn{2}{|c|}{$70.6(39-91)$} \\
\hline Lymphocytes & \multicolumn{2}{|c|}{$21.7(3.6-50.5)$} & \multicolumn{2}{|c|}{$21.4(6-47.1)$} \\
\hline Eosinophils & \multicolumn{2}{|c|}{$0.2(0-2)$} & \multicolumn{2}{|c|}{$0.3(0-1.5)$} \\
\hline Basophils & \multicolumn{2}{|c|}{$1.0(0-13.8)$} & \multicolumn{2}{|c|}{$1.0(0-11.6)$} \\
\hline Monocytes & \multicolumn{2}{|c|}{$6.1(1.7-15)$} & \multicolumn{2}{|c|}{$6.0(2-17)$} \\
\hline Neutrophil to lymphocyte ratio & \multicolumn{2}{|c|}{$3.2(0.7-26.1)$} & \multicolumn{2}{|c|}{$3.3(0.9-15.2)$} \\
\hline
\end{tabular}

ECOG, Eastern Cooperative Oncology Group; PPV, personalized peptide vaccination; PSA, prostate-specific antigen; WBC, white blood cell.

withdrawal of consent for treatment (3.4 vs. 2.9\%). After the study treatment, 148 of 204 patients $(72.5 \%)$ in the PPV arm and 71 of 102 patients $(69.6 \%)$ in the placebo arm used enzalutamide or abiraterone.

For OS, there were 160 deaths (78.4\%) in the PPV arm and $77(75.5 \%)$ in the placebo arm. PPV did not improve OS compared with placebo (median OS, 16.1 months $(95 \% \mathrm{CI}$, 13-18.2 months) with PPV and 16.9 months (95\% CI, 13.1-20.4 months) with placebo; HR, 1.04; 95\% CI, 0.80-1.37; $\mathrm{P}=0.77$; Fig. 2A). The estimated median PFS was 4.2 months (95\% CI, 4.0-5.6 months) with PPV and 5.8 months (95\% CI, 4.1-7.3 months) with placebo (HR, 1.09; 95\% CI, 0.85-1.40; $\mathrm{P}=0.32$; Fig. 2B). The median one-year survival rate was also similar in the two arms (62.3 vs. $62.4 \%)$.
For immune responses, pre-treatment plasma and peripheral blood mononuclear cell (PBMC) samples were available from all 306 patients. The mean peptide-specific IgG and CTL levels to the selected peptides were 154 fluorescence intensity units (FIU) and $0.7 \mathrm{pg} / \mathrm{ml}$ in the PPV arm, and 311 FIU and $0.8 \mathrm{pg} / \mathrm{ml}$ in the placebo arm, respectively. After the 6-dose treatment, 189 samples from the PPV arm and 96 samples from the placebo arm were available for immune response analysis, and the mean values of IgG and CTL were significantly higher in the PPV arm than in the placebo arm (9716 vs. 284 FIU for IgG responses; $\mathrm{P}<0.01 ; 80.0$ vs. $0.7 \mathrm{pg} / \mathrm{ml}$ for CTL activity; $\mathrm{P}<0.01)$. Significant peptide-specific IgG and CTL increases were observed in 27.5 and $55.6 \%$ of the 189 PPV patients, respectively, but no increases were observed in the placebo 
A

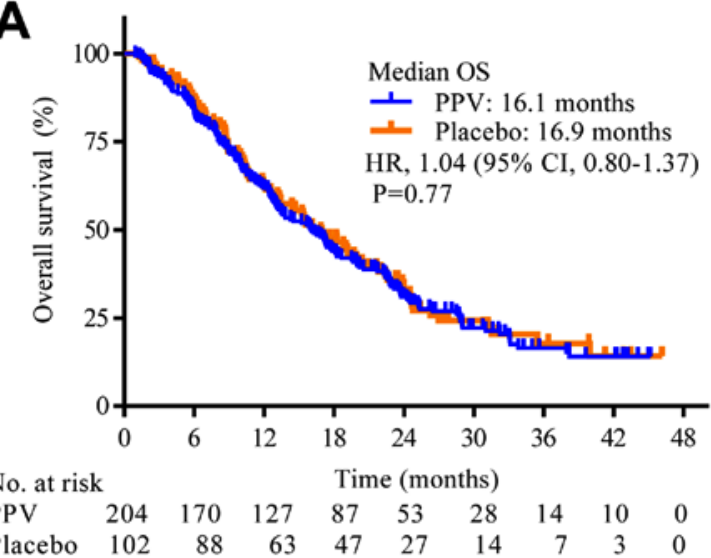

B

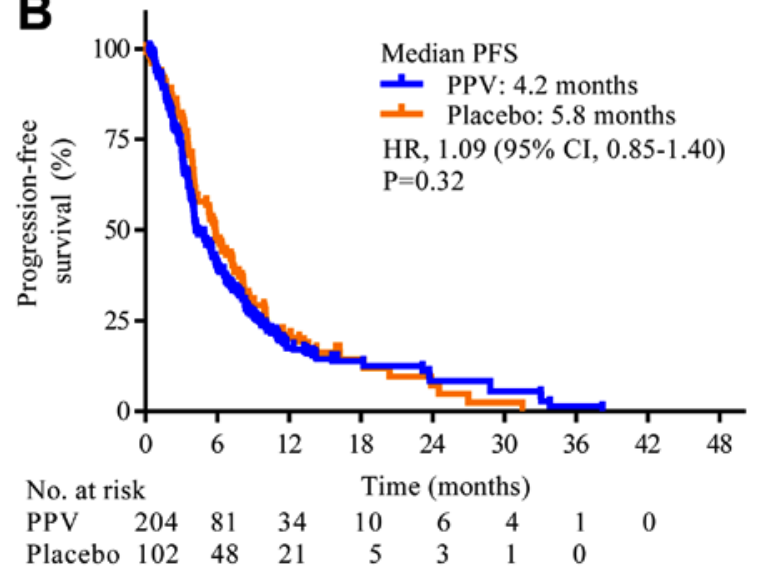

Figure 2. Kaplan-Meier curves showing no difference in (A) overall survival and (B) progression-free survival between PPV and placebo. PPV, personalized peptide vaccination.

patients throughout the study period. There was no relationship among IgG, CTL, and OS in the PPV arm following application of the linear regression model [Fig. S1A; $\mathrm{R}^{2}=0.00012$, between $\operatorname{IgG}$ change and $\mathrm{OS}$ after the 6-dose treatment, and $\mathrm{R}^{2}=0.0026$, between CTL change and OS after the 6-dose treatment (Fig. S1B)].

To further investigate the effects of PPV, we evaluated treatment arm effects among patient subgroups. Initially, we separated subgroups by the subsets listed in Table I. This analysis revealed lower HRs for OS in favor of the PPV arm in patients with $<$ the median\% neutrophils or $\geq$ the median\% lymphocytes among white blood cell types at baseline (HR, 0.82; 95\% CI, 0.56-1.23 for <median\% neutrophil or HR, 0.80; 95\% CI, 0.54-1.19 for $\geq$ median\% lymphocyte) by the significant interaction test $(\mathrm{P}=0.04$ or $\mathrm{P}=0.05$, respectively; Fig. 3). Based on this unexpected interaction among $\%$ neutrophils, \% lymphocytes, and the efficacy of PPV, we analyzed the most relevant \% neutrophil or \% lymphocyte cut-off. We plotted interaction P-values from neutrophil proportions of $50-80 \%$ (median value, $70 \%$ ) or those from lymphocyte proportions of $10-40 \%$ (median value, $21.6 \%$ ) and the number of target patients at each point (Fig. S1). The most relevant $\%$ neutrophil and \% lymphocyte cut-offs were 64 and $26 \%$, respectively, with an interaction of $\mathrm{P}<0.01$ and a larger number of patients (Fig. S2). We reanalyzed treatment arm effects using the cut-off of 64\% neutrophils or 26\% lymphocytes, and found lower HRs for OS in PPV arm patients (HR, 0.55; 95\% CI, 0.33-0.93 for <64\% neutrophils or HR, 0.70; 95\% CI, 0.52-0.92 for $\geq 26 \%$ lymphocytes) than in the initial analysis using the median cut-off with an interaction $\mathrm{P}=0.003$ or $\mathrm{P}=0.007$, respectively (Fig. 3 ). This interaction among $64 \%$ neutrophils, $26 \%$ lymphocytes, and the PPV treatment is shown in Fig. 4. The median OS in the PPV arm of patients with $<64 \%$ neutrophils or $\geq 26 \%$ lymphocytes was significantly longer than that in the placebo arm of patients in the same subgroups (median OS, 22.9 vs. 15.4 months (Fig. 4A); $\mathrm{P}=0.03$ or median OS, 23.3 vs. 13.8 months; $\mathrm{P}=0.02$, respectively; Fig. 4B). By contrast, the median OS in the PPV arm of patients with $\geq 64 \%$ neutrophils or with $<26 \%$ lymphocytes was not different from that in the placebo arm of patients in the same subgroups (Fig. 4C and D). On analysis of immune responses for these factors, IgG changes in the PPV arm of patients with $<64 \%$ neutrophils or $\geq 26 \%$ lymphocytes during the first 6 doses were significantly higher than those in patients in the complementary subgroup $(\mathrm{P}=0.02$ or $\mathrm{P}=0.01$, respectively; t-test; Fig. 4E), but CTL changes in these subgroups were not significantly different $(\mathrm{P}=0.64$ or $\mathrm{P}=0.27$, respectively; t-test; Fig. 4F).

All AEs related to the study treatment (peptide plus adjuvant or placebo plus adjuvant) are shown in Table II. Injection site reactions and nausea were more frequent with PPV ( 86.8 vs. $74.5 \%$ and 9.3 vs. $2.9 \%$, respectively). The most common AEs were $<$ grade 3 injection site reactions in both arms. Treatment-related AEs of $\geq$ grade 3 were observed in 83 patients in the PPV arm (40.7\%) and 42 patients in the placebo arm (41.2\%).

\section{Discussion}

Peptide-based vaccines are designed to elicit CTL against antigens selectively expressed by tumor cells. PPV, in which a maximum of 4 HLA class IA-matched peptides are selected for vaccination from a pool of peptides based on both HLA class IA type and the pre-existing host immunity before vaccination, are designed to stimulate antigen-specific memory $\mathrm{T}$ cells (11). In a trial of neoadjuvant peptide vaccination before radical prostatectomy for patients with localized prostate cancer, we previously reported that PPV quickly induced the infiltration of $\mathrm{CD} 45 \mathrm{RO}+$ memory $\mathrm{T}$ cells, rather than naïve T or B cells, into cancer tissues (17). In previous phase II trials, PPV was demonstrated to improve OS in chemotherapy-naïve patients with CRPC or in patients with docetaxel-resistant CRPC (12-14). The only approved cancer vaccine for CRPC is sipuleucel-T (autologous dendritic-cell vaccine). This cancer vaccine is considered for patients with less advanced disease who may benefit from sipuleucel-T treatment, providing rationale for immunotherapy as an early treatment strategy in patients with CRPC (18). This phase III randomized trial investigated PPV as a second-line treatment after docetaxel chemotherapy in patients with progressing CRPC. To the best of our knowledge, this is the first trial addressing this strategy in a relatively large number of patients for whom docetaxel induction chemotherapy failed. However, the present study demonstrated no difference in OS or PFS between PPV and placebo. 


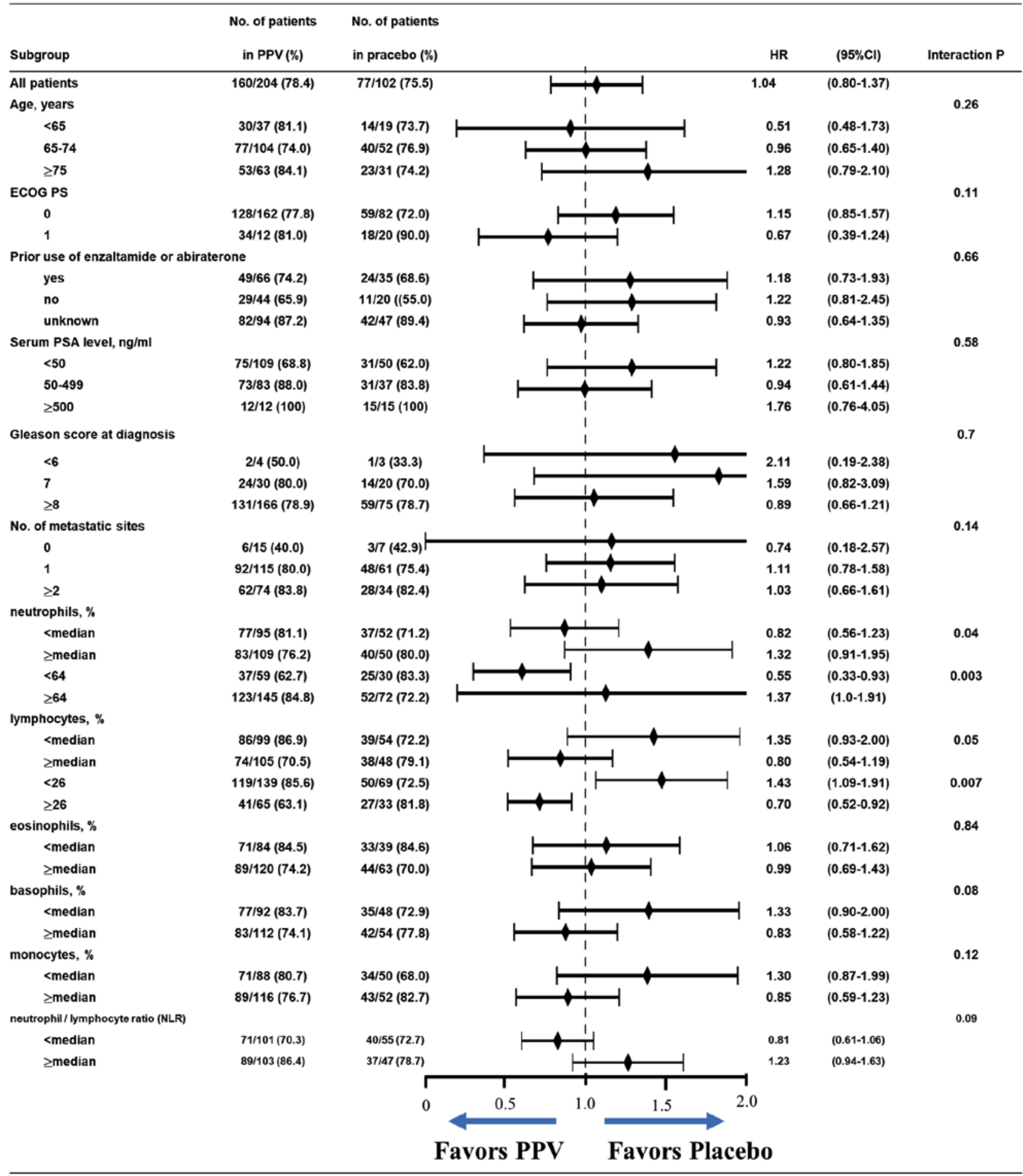

Figure 3. Forest plot showing hazards ratios and 95\% confidence intervals for overall survival time for subgroup analysis of stratification factors (full analysis set population). ECOG, Eastern Cooperative Oncology Group; HR, hazard ratio; PPV, personalized peptide vaccination; PS, performance status; PSA, prostate-specific antigen.

There are several explanations for the lack of OS or PFS improvement in this study. First, the target patients may have had heterogeneous immune cell repertoires, a large tumor burden, and many immune suppressive elements, such as increased myeloid-derived suppressor cells (MDSC) or regulatory $\mathrm{T}$ cells in the tumor microenvironment, and it is well known that tumor-associated immunosuppression is significantly involved in tumor progression and resistance to immunotherapy $(19,20)$. Second, efficiently primed $\mathrm{T}$ cells may lose their responsiveness to tumor antigens. This may be explained by the downregulation or loss of tumor antigens and T-cell inhibition mediated by checkpoint molecules such as CTLA-4, PD-1 and PDL-1 (21,22). Third, another contributory factor may be the availability of more effective salvage therapies that prolong OS after the study treatment, many of which were not widely available at the time of the previous phase II study of PPV. The current availability of such drugs (e.g., cabazitaxel, abiraterone, enzalutamide, and radium-223) 
A
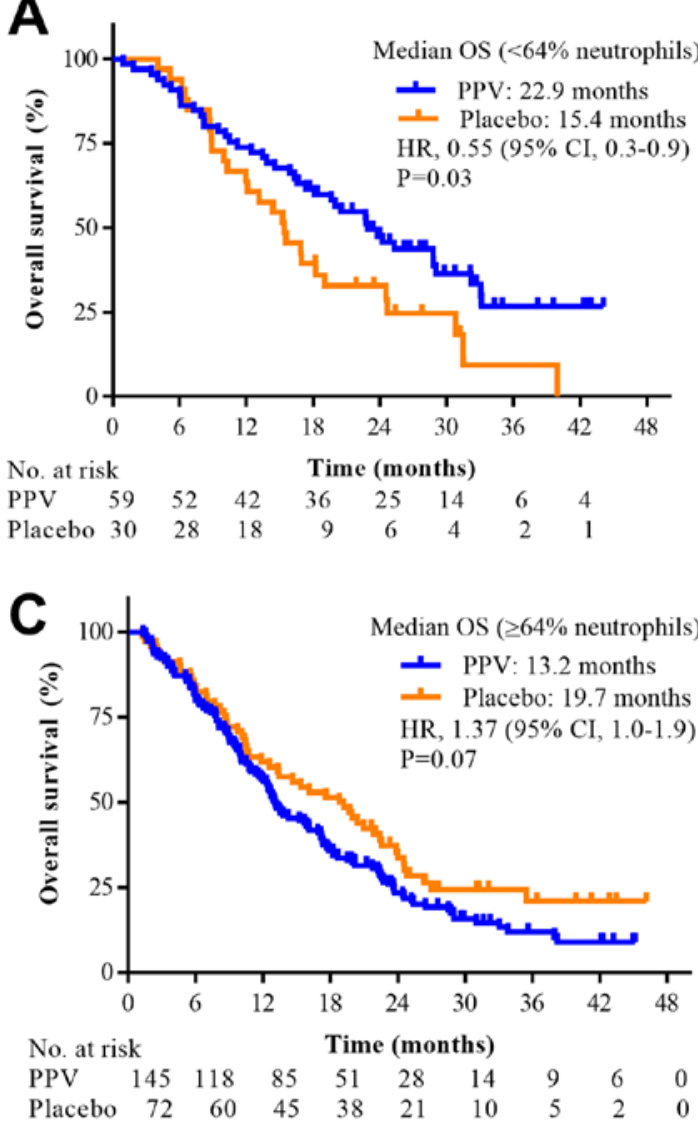

E

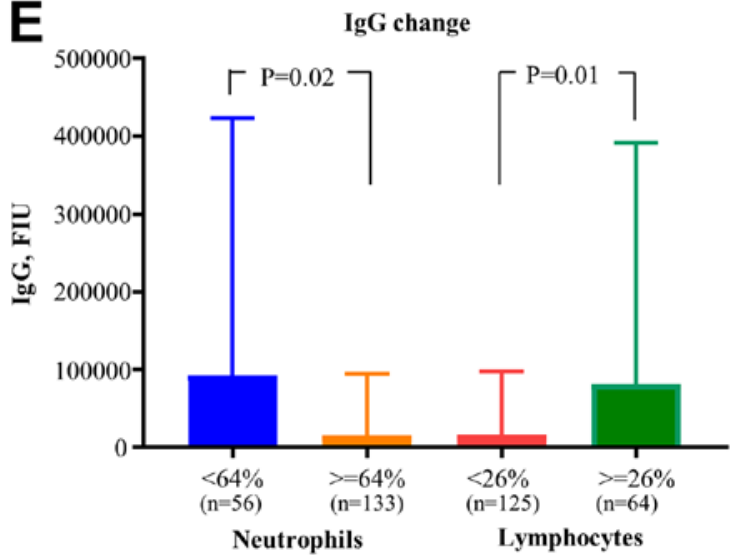

B
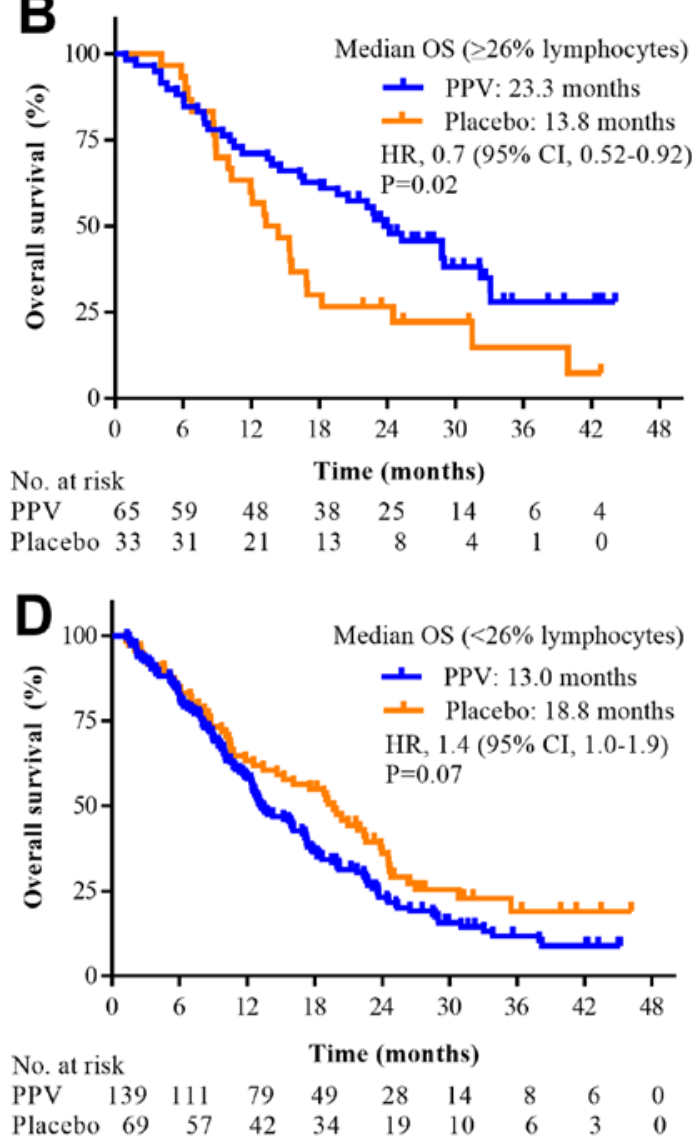

$\mathbf{F}$

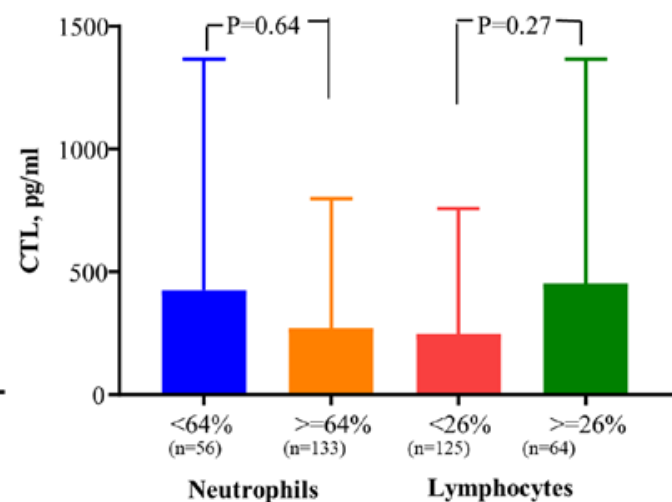

Figure 4. Comparison of treatment effects. (A) OS in patients with $<64 \%$ neutrophils according to PPV versus placebo treatment. (B) OS in patients with $\geq 26 \%$ lymphocytes according to PPV versus placebo treatment. (C) OS in patients with $\geq 64 \%$ neutrophils according to PPV versus placebo treatment. (D) OS in patients with $<26 \%$ lymphocytes according to PPV versus placebo treatment. (E) IgG changes from baseline to 6 doses in the PPV arm of patients according to neutrophil and lymphocyte proportions. (F) CTL changes from baseline to 6 doses in the PPV arm of patients according to neutrophil proportion. OS, overall survival; PPV, personalized peptide vaccination; CTL, cytotoxic T lymphocyte; FIU, fluorescence intensity units; IgG, immunoglobulin G; HR, hazard ratio.

may have affected the disease course in patients receiving PPV or placebo. Fourth, the treatment was discontinued early before sufficient doses of PPV were administered, thereby affecting the efficacy due to the lack of notable objective responses or PSA responses.

Further studies of predictive biomarkers of PPV efficacy may be necessary to determine whether subgroups may improve the OS. The recent discovery that cancers deficient in DNA mismatch-repair function (dMMR) or with microsatellite instability (MSI-high) demonstrate high rates of objective tumor responses to immune checkpoint therapies (23) led to the FDA approval of pembrolizumab for the treatment of advanced dMMR/MSH-high cancers of any histological type, among which mCRPC patients are a small subset. Previous findings showed that the abnormal granulocytes present in the PBMC fraction at baseline may lead to the poor prognosis of advanced prostate cancer patients receiving PPV treatment using DNA microarray analysis (24). In addition, the increase in granulocytic MDSC after PPV treatment was an unfavorable marker for the OS of mCRPC patients (25). Those results suggested that the proportion of neutrophils, the majority of granulocytes, affects the efficacy of PPV 
Table II. Any grade or severe AEs recorded in patients by treatment arm (CTCAE version 4.0).

\begin{tabular}{|c|c|c|c|c|c|c|c|c|c|c|}
\hline \multirow[b]{2}{*}{$\mathrm{AE}^{\mathrm{a}}$} & \multicolumn{2}{|c|}{$\begin{array}{c}\text { PPV } \\
(n=204) \\
\text { Any grade }\end{array}$} & \multicolumn{2}{|c|}{$\begin{array}{c}\text { Placebo } \\
(n=102) \\
\text { Any grade }\end{array}$} & \multirow[b]{2}{*}{ P-value ${ }^{b}$} & \multicolumn{2}{|c|}{$\begin{array}{c}\text { PPV } \\
(n=204) \\
\text { Grade } \geq 3\end{array}$} & \multicolumn{2}{|c|}{$\begin{array}{c}\text { Placebo } \\
(n=102) \\
\text { Grade } \geq 3\end{array}$} & \multirow[b]{2}{*}{ P-value } \\
\hline & No. & $\%$ & No. & $\%$ & & No. & $\%$ & No. & $\%$ & \\
\hline All AEs & 178 & 87.3 & 79 & 77.5 & 0.02 & 83 & 40.7 & 42 & 41.2 & 0.52 \\
\hline Injection site reaction & 177 & 86.8 & 76 & 74.5 & 0.008 & 5 & 0.0 & 1 & 0.0 & 0.35 \\
\hline Cancer pain & 54 & 26.5 & 22 & 21.6 & 0.21 & 18 & 8.8 & 9 & 8.8 & 0.59 \\
\hline Decreased appetite & 37 & 18.1 & 13 & 12.7 & 0.15 & 19 & 9.3 & 5 & 4.9 & 0.13 \\
\hline Edema peripheral & 35 & 17.2 & 13 & 12.7 & 0.20 & 1 & 0.5 & 2 & 2.0 & 1.0 \\
\hline Pyrexia & 31 & 15.2 & 12 & 11.8 & 0.26 & 2 & 1.0 & 0 & 0.0 & 0.45 \\
\hline Anemia & 29 & 14.2 & 20 & 19.6 & 0.91 & 21 & 10.3 & 12 & 11.8 & 0.72 \\
\hline Constipation & 29 & 14.2 & 14 & 13.7 & 0.53 & 1 & 0.5 & 1 & 1.0 & 0.89 \\
\hline Nasopharyngitis & 28 & 13.7 & 9 & 8.8 & 0.15 & 0 & 0.0 & 0 & 0.0 & n.a. \\
\hline Malaise & 28 & 13.7 & 9 & 8.8 & 0.15 & 2 & 1.0 & 1 & 1.0 & 0.74 \\
\hline Weight gain & 22 & 10.8 & 12 & 11.8 & 0.68 & 2 & 1.0 & 1 & 1.0 & 0.74 \\
\hline Nausea & 19 & 9.3 & 3 & 2.9 & 0.03 & 3 & 1.5 & 1 & 1.0 & 0.59 \\
\hline Subcutaneous hemorrhage & 19 & 9.3 & 20 & 19.6 & 1.0 & 0 & 0.0 & 0 & 0.0 & n.a. \\
\hline Fall & 17 & 8.3 & 5 & 4.9 & 0.20 & 0 & 0.0 & 1 & 1.0 & 1.0 \\
\hline Diabetes mellitus & 16 & 7.8 & 7 & 6.9 & 0.48 & 4 & 2.0 & 2 & 2.0 & 0.68 \\
\hline Diarrhea & 16 & 7.8 & 8 & 7.8 & 0.60 & 1 & 0.5 & 0 & 0.0 & 0.67 \\
\hline Prostate cancer & 15 & 7.4 & 4 & 3.9 & 0.18 & 15 & 7.4 & 4 & 3.9 & 0.18 \\
\hline Hypertension & 15 & 7.4 & 6 & 5.9 & 0.41 & 10 & 4.9 & 5 & 4.9 & 0.62 \\
\hline Vomiting & 15 & 7.4 & 7 & 6.9 & 0.54 & 1 & 0.5 & 1 & 1.0 & 0.90 \\
\hline Back pain & 15 & 7.4 & 6 & 5.9 & 0.41 & 2 & 1.0 & 1 & 1.0 & 0.74 \\
\hline Insomnia & 14 & 6.9 & 5 & 4.9 & 0.35 & 0 & 0.0 & 0 & 0.0 & n.a. \\
\hline Weight loss & 12 & 5.9 & 9 & 8.8 & 0.88 & 1 & 0.5 & 1 & 1.0 & 0.90 \\
\hline Abnormal hepatic function & 11 & 5.4 & 2 & 2.0 & 0.13 & 4 & 2.0 & 0 & 0.0 & 0.20 \\
\hline Urinary tract infection & 10 & 4.9 & 7 & 6.9 & 0.83 & 3 & 1.5 & 1 & 1.0 & 0.59 \\
\hline Urinary retention & 9 & 4.4 & 6 & 5.9 & 0.92 & 1 & 0.5 & 1 & 1.0 & 0.90 \\
\hline Hydronephrosis & 8 & 3.9 & 7 & 6.9 & 0.92 & 4 & 2.0 & 3 & 2.9 & 0.83 \\
\hline Platelet count reduction & 8 & 3.9 & 9 & 8.8 & 0.80 & 5 & 2.5 & 2 & 2.0 & 0.57 \\
\hline Hematuria & 7 & 3.4 & 6 & 5.9 & 0.90 & 3 & 1.5 & 2 & 2.0 & 0.79 \\
\hline Dental caries & 3 & 1.5 & 6 & 5.9 & 0.99 & 1 & 0.5 & 0 & 0.0 & 0.67 \\
\hline
\end{tabular}

${ }^{a}$ Medical Dictionary for Regulatory Activities, version 20.0 (MedDRA) terms; ${ }^{b}$ Fisher's exact test. AE, adverse event; CTCAE, Common Terminology Criteria for Adverse Events; n.a., not available; PPV, personalized peptide vaccination.

treatment. The post hoc analysis in this trial revealed that patients with a low neutrophil proportion $(<64 \%)$ or a high lymphocyte proportion ( $\geq 26 \%$ ) at baseline in the PPV arm had a significantly longer OS than their counterparts in the placebo arm; however, the proportions of eosinophils, basophils, and monocytes did not affect the efficacy of PPV treatment even though the neutrophil to lymphocyte ratio (NLR) was used. Although NLR was reported as a risk factor for OS of patients with mCRPC, similar to many other advanced cancers, when it was higher than 2 (26-28), we found it to be less sensitive than the proportion of neutrophils or lymphocytes as a biomarker to predict the efficacy of PPV with an interaction $\mathrm{P}=0.09$. This discrepancy may have occurred, in part, because the NLR value as a risk factor was based on a comparison of the lower and higher levels among patients in the same treatment arm in the previous studies rather than between patients in different treatment arms (i.e., PPV and placebo). The levels of PPV-induced IgG were significantly higher in patients with $<64 \%$ neutrophils or $\geq 26 \%$ lymphocytes treated by PPV than in their counterparts, and the median OS of these groups was significantly longer than that of the counter groups. This suggested that patients with $<64 \%$ neutrophils or $\geq 26 \%$ lymphocytes can receive survival benefits from PPV treatment. Although the reason for the baseline neutrophil and lymphocyte proportions most strongly affecting the clinical benefits is presently unknown, the following hypothesis was considered: Inflammatory responses associated with tumor are considered to be one of the major events to escape from immune attack and promote the production of inflammatory cytokines. These responses result in the circulation of a few 
lymphocytes along with many neutrophils. Therefore, the baseline neutrophil and lymphocyte proportions may be one of the keys for successful immune induction (29-31).

The tolerability of PPV treatment was good overall and the most common treatment-related $\mathrm{AE}$ in both arms was injection site reaction, which was mainly caused by incomplete Freund's adjuvant (32). Dose interruptions or reductions were infrequent, and the overall safety profile was consistent with that observed in previous phase II trials. Treatment-related deaths were not increased by PPV, suggesting a lack of toxicity as the main contributing factor.

In conclusion, PPV did not prolong OS or PFS in HLA-A24-positive patients with CRPC progressing after docetaxel chemotherapy. Subgroup analyses demonstrated that patients with a low neutrophil proportion or a high lymphocyte proportion at baseline in the PPV arm had a significantly longer OS than their counterparts in the placebo arm in this setting. Additional confirmation of this finding is required to better define subgroups of patients who can receive PPV treatment for progressive CRPC after docetaxel chemotherapy.

\section{Acknowledgements}

Not applicable.

\section{Funding}

This study was supported by grants from the Japan Agency for Medical Research and Development (no. 18im0110802h0008) and Fujifilm Company. The funders of the study had no role in the study design, data collection, data analysis, data interpretation, or writing of the report.

\section{Availability of data and materials}

The datasets used and/or analyzed during the current study are available from the corresponding author on reasonable request.

\section{Author's contribution}

MN, HU, SN, SE, HF and KI contributed to the conception and design of the study. KF, GA, HU, KH, HM, SF, YK, HN, AT, MF, SN, SE and HF provided study materials or patients. $\mathrm{MN}$ and $\mathrm{SH}$ completed the statistical analyses. MN reviewed the clinical data. All authors interpreted data and reviewed and approved the final article.

\section{Ethics approval and consent to participate}

This study was approved by the Ethics Committees of Kurume University (approval no. 213019) and each institution, and it was registered in the UMIN Clinical Trials Registry (no. UMIN000011308) and the Pharmaceuticals and Medical Devices Agency in Japan (no. 25-0917). The study was carried out in accordance with the Declaration of Helsinki and the International Conference on Harmonization of Good Clinical Practice guidelines. Written informed consent to participate in the clinical trial and to use their data for research and publication purpose was received from all individual participants before participating in the study.

\section{Patient consent for publication}

Not applicable.

\section{Competing interests}

MN served as an advisory board consultant for BrightPath Biotherapeutics Co., Ltd. KI received research funding from Taiho Pharmaceutical Company. SN served as a consultant to BrightPath Biotherapeutics Co., Ltd and received honorarium from Sanofi. All other authors declare no competing interests.

\section{References}

1. Tannock IF, de Wit R, Berry WR, Horti J, Pluzanska A, Chi KN Oudard S, Théodore C, James ND, Turesson I, et al; TAX 327 Investigators: Docetaxel plus prednisone or mitoxantrone plus prednisone for advanced prostate cancer. N Engl J Med 351: 1502-1512, 2004.

2. Berthold DR, Pond GR, Soban F, de Wit R, Eisenberger M and Tannock IF: Docetaxel plus prednisone or mitoxantrone plus prednisone for advanced prostate cancer: Updated survival in the TAX 327 study. J Clin Oncol 26: 242-245, 2008.

3. de Bono JS, Oudard S, Ozguroglu M, Hansen S, Machiels JP, Kocak I, Gravis G, Bodrogi I, Mackenzie MJ, Shen L, et al; TROPIC Investigators: Prednisone plus cabazitaxel or mitoxantrone for metastatic castration-resistant prostate cancer progressing after docetaxel treatment: A randomised open-label trial. Lancet 376: 1147-1154, 2010.

4. De Bono JS, Logothetis CJ, Molina A, Fizazi K, North S, Chu L, Chi KN, Jones RJ, Goodman OB Jr, Saad F, et al: Abiraterone and increased survival in mPrCa. N Engl J Med 354: 1995-2005, 2011.

5. Scher HI, Fizazi K, Saad F, Taplin ME, Sternberg CN, Miller K, de Wit R, Mulders P, Chi KN, Shore ND, et al; AFFIRM Investigators: Increased survival with enzalutamide in prostate cancer after chemotherapy. N Engl J Med 367: 1187-1197, 2012.

6. Parker C, Nilsson S, Heinrich D, Helle SI, O'Sullivan JM, Fosså SD, Chodacki A, Wiechno P, Logue J, Seke M, et al; ALSYMPCA Investigators: Alpha emitter radium-223 and survival in metastatic prostate cancer. N Engl J Med 369: 213-223, 2013.

7. Kantoff PW, Higano CS, Shore ND, Berger ER, Small EJ, Penson DF, Redfern CH, Ferrari AC, Dreicer R, Sims RB, et al; IMPACT Study Investigators: Sipuleucel-T immunotherapy for castration-resistant prostate cancer. N Engl J Med 363: 411-422, 2010.

8. Isaacsson Velho P and Antonarakis ES: PD-1/PD-L1 pathway inhibitors in advanced prostate cancer. Expert Rev Clin Pharmacol 11: 475-486, 2018.

9. Kwon ED, Drake CG, Scher HI, Fizazi K, Bossi A, van den Eertwegh AJ, Krainer M, Houede N, Santos R, Mahammedi H, et al; CA184-043 Investigators: Ipilimumab versus placebo after radiotherapy in patients with metastatic castration-resistant prostate cancer that had progressed after docetaxel chemotherapy (CA184-043): A multicentre, randomised, double-blind, phase 3 trial. Lancet Oncol 15: 700-712, 2014.

10. Nuhn P, De Bono JS, Fizazi K, Freedland SJ, Grilli M, Kantoff PW, Sonpavde G, Sternberg CN, Yegnasubramanian S and Antonarakis ES: Update on systemic prostate cancer therapies: Management of metastatic castration-resistant prostate cancer in the era of precision oncology. Eur Urol 75: 88-99, 2019.

11. Noguchi M, Sasada T and Itoh K: Personalized peptide vaccination: A new approach for advanced cancer as therapeutic cancer vaccine. Cancer Immunol Immunother 62: 919-929, 2013.

12. Noguchi M, Uemura H, Naito S, Akaza H, Yamada A and Itoh K: A phase I study of personalized peptide vaccination using 14 kinds of vaccine in combination with low-dose estramustine in HLA-A24-positive patients with castration-resistant prostate cancer. Prostate 71: 470-479, 2011.

13. Yoshimura K, Minami T, Nozawa M, Kimura T, Egawa S, Fujimoto H, Yamada A, Itoh $\mathrm{K}$ and Uemura $\mathrm{H}$ : A phase 2 randomized controlled trial of personalized peptide vaccine immunotherapy with low-dose dexamethasone versus dexamethasone alone in chemotherapy-naïve castration-resistant prostate cancer. Eur Urol 70: 35-41, 2016. 
14. Noguchi M, Moriya F, Suekane S, Matsuoka K, Arai G, Matsueda S, Sasada T, Yamada A and Itoh K: Phase II study of personalized peptide vaccination for castration-resistant prostate cancer patients who failed in docetaxel-based chemotherapy. Prostate 72: 834-845, 2012.

15. Komatsu N, Shichijo S, Nakagawa $M$ and Itoh K: New multiplexed flow cytometric assay to measure anti-peptide antibody: A novel tool for monitoring immune responses to peptides used for immunization. Scand J Clin Lab Invest 64: 535-545, 2004.

16. Hida N, Maeda Y, Katagiri K, Takasu H, Harada M and Itoh K A simple culture protocol to detect peptide-specific cytotoxic $\mathrm{T}$ lymphocyte precursors in the circulation. Cancer Immuno Immunother 51: 219-228, 2002.

17. Noguchi M, Yao A, Harada M, Nakashima O, Komohara Y, Yamada S, Itoh K and Matsuoka K: Immunological evaluation of neoadjuvant peptide vaccination before radical prostatectomy for patients with localized prostate cancer. Prostate 67: 933-942, 2007.

18. Schellhammer PF, Chodak G, Whitmore JB, Sims R, Frohlich MW and Kantoff PW: Lower baseline prostate-specific antigen is associated with a greater overall survival benefit from sipuleucel-T in the Immunotherapy for Prostate Adenocarcinoma Treatment (IMPACT) trial. Urology 81: 1297-1302, 2013.

19. Gabrilovich DI, Bronte V, Chen SH, Colombo MP, Ochoa A, Ostrand-Rosenberg S and Schreiber H: The terminology issue for myeloid-derived suppressor cells. Cancer Res 67: 425-426, author reply 426, 2007.

20. Miller AM, Lundberg K, Ozenci V, Banham AH, Hellström M, Egevad L and Pisa P: CD4+CD25high T cells are enriched in the tumor and peripheral blood of prostate cancer patients. J Immunol 177: 7398-7405, 2006.

21. Topalian SL, Hodi FS, Brahmer JR, Gettinger SN, Smith DC, McDermott DF, Powderly JD, Carvajal RD, Sosman JA, Atkins MB, et al: Safety, activity, and immune correlates of anti-PD-1 antibody in cancer. N Engl J Med 366: 2443-2454, 2012.

22. Anderson MJ, Shafer-Weaver K, Greenberg NM and Hurwitz AA: Tolerization of tumor-specific $\mathrm{T}$ cells despite efficient initial priming in a primary murine model of prostate cancer. J Immunol 178: 1268-1276, 2007.
23. Le DT, Durham JN, Smith KN, Wang H, Bartlett BR, Aulakh LK, Lu S, Kemberling H, Wilt C, Luber BS, et al: Mismatch repair deficiency predicts response of solid tumors to PD-1 blockade. Science 357: 409-413, 2017.

24. Komatsu N, Matsueda S, Tashiro K, Ioji T, Shichijo S, Noguchi M, Yamada A, Doi A, Suekane S, Moriya F, et al: Gene expression profiles in peripheral blood as a biomarker in cancer patients receiving peptide vaccination. Cancer 118: 3208-3221, 2012.

25. Noguchi M, Moriya F, Koga N, Matsueda S, Sasada T, Yamada A, Kakuma T and Itoh K: A randomized phase II clinical trial of personalized peptide vaccination with metronomic low-dose cyclophosphamide in patients with metastatic castration-resistant prostate cancer. Cancer Immunol Immunother 65: 151-160, 2016.

26. Coffelt SB, Wellenstein MD and de Visser KE: Neutrophils in cancer: Neutral no more. Nat Rev Cancer 16: 431-446, 2016.

27. Shaul ME and Fridlender ZG: Cancer-related circulating and tumor-associated neutrophils - subtypes, sources and function. FEBS J 285: 4316-4342, 2018.

28. Dolan RD, Laird BJA, Horgan PG and McMillan DC: The prognostic value of the systemic inflammatory response in randomised clinical trials in cancer: A systematic review. Crit Rev Oncol Hematol 132: 130-137, 2018.

29. Dvorak HF: Tumors: Wounds that do not heal-redux. Cancer Immunol Res 3: 1-11, 2015.

30. Steinman RM, Hawiger D and Nussenzweig MC: Tolerogenic dendritic cells. Annu Rev Immunol 21: 685-711, 2003.

31. Basile D, Garattini SK, Bonotto M, Ongaro E, Casagrande M, Cattaneo M, Fanotto V, De Carlo E, Loupakis F, Urbano F, et al: Immunotherapy for colorectal cancer: Where are we heading? Expert Opin Biol Ther 17: 709-721, 2017.

32. van Doorn E, Liu H, Huckriede A and Hak E: Safety and tolerability evaluation of the use of Montanide ISA ${ }^{\mathrm{TM}} 51$ as vaccine adjuvant: A systematic review. Hum Vaccin Immunother 12: 159-169, 2016

(i) $(5)$ This work is licensed under a Creative Commons Attribution-NonCommercial-NoDerivatives 4.0 International (CC BY-NC-ND 4.0) License. 